\title{
Uso de influencers en el marketing digital de las empresas turísticas españolas
}

\section{Use of influencers in the Spanish tourism companies' digital marketing}

Uso de influenciadores no marketing digital das empresas de turismo espanholas

Paula Rodríguez, Universidad de Santiago de Compostela, Santiago de Compostela, España (paula.rodriguezvalderrey@sitel.com)

José Sixto García, Universidad de Santiago de Compostela, Santiago de Compostela, España (jose.sixto@usc.es)

RESUMEN | El marketing de influencia es un fenómeno en auge. Cada vez más organizaciones recurren a influencers para comunicar su mensaje a través de las redes sociales, sobre todo en Instagram y YouTube. En este trabajo analizamos el uso que hacen de los influencers las diez empresas turísticas españolas con mayor inversión publicitaria durante 2019 en esas dos redes sociales. Se emplea una metodología de modelo mixto para analizar la utilización de este tipo de figuras en la comunicación de las entidades, detectar los tipos de perfiles profesionales a los que se recurre y determinar si su empleo aumenta el valor de las cuentas organizacionales. Los resultados evidencian preferencia por Instagram frente a YouTube, un aumento del Earned Media Value, y que 60\% de las empresas analizadas recurren a influencers en su comunicación habitual, aunque apuestan por especialistas y no necesariamente a perfiles con un número mayor de seguidores.

PALABRAS CLAVE: marketing de influencia; Instagram; YouTube; influencer; turismo. 
ABSTRACT | Influencer marketing is a booming tool. More and more organizations are turning to influencers to communicate their message through social networks, especially on Instagram and YouTube. In this research, we analyze the use made of influencers by the Spanish tourism companies that invested the most in advertising during 2019 in these two social networks. A mixed model methodology is used to analyze the use of this type of figures in the communication of these entities, detect the types of professional profiles used, and determine whether their employment increases the value of organizational accounts. The results show a preference for Instagram over YouTube, an increase in Earned Media Value and that $60 \%$ of the companies analyzed are already turning to influencers in their regular communication, although they are betting on specialists whether than in profiles with a larger number of followers.

KEYWORDS: influencer marketing; Instagram; YouTube; influencer; tourism.

RESUMO | O marketing de influenciadores é um fenômeno em expansão. Cada vez mais organizações estão recorrendo a influenciadores para comunicar sua mensagem por meio de redes sociais, especialmente através do Instagram e do YouTube. Neste trabalho analisamos a utilização de influenciadores nestas duas redes sociais pelas dez empresas de turismo espanholas com maior investimento publicitário durante 2019. Uma metodologia de modelo misto é utilizada para analisar o uso deste tipo de figuras na comunicação das entidades, detetar os tipos de perfis profissionais que são utilizados e determinar se seu emprego aumenta o valor das contas organizacionais. Os resultados mostram uma preferência pelo Instagram em relação ao YouTube, um aumento do valor mídia ganho e que o 60\% das entidades analisadas já está se voltando para influenciadores na sua comunicação regular, embora optem por especialistas do setor em relação a perfis com maior número de seguidores.

PALAVRAS-CHAVE: marketing de influência; Instagram; YouTube; influenciador; turismo. 


\section{INTRODUCCIÓN}

Pese a su historia relativamente corta, el marketing digital ha sufrido grandes cambios debido, principalmente, a la heterogeneidad de sus canales, propiciada por la continua evolución de Internet. Nos encontramos en una continua experimentación de un entorno en el que el marketing cambia sin cesar, evoluciona y se transforma (ESIC, 2018). Algunas de las tendencias de marketing digital con más auge en la actualidad son 1) el inbound marketing (Galindo, 2018), que combina técnicas no intrusivas para captar clientes atraídos por productos que les reportan valor; 2) el marketing de contenidos (Núñez, 2018), para entender exactamente qué necesitan los usuarios, con el objetivo de ofrecérselo de la forma más convincente posible; 3) el email marketing (Rentero, 2018), estrategia basada en el envío de correos electrónicos a un usuario que ha dado su consentimiento previo demostrando su interés en la marca; 4) el marketing de buscadores (Key, 2017) o SEM, consistente en obtener tráfico mediante la compra de anuncios en buscadores como Google o Yahoo, o 5) el marketing móvil (Sixto-García et al., 2021), que potencia las cualidades del teléfono móvil -por ejemplo, la geolocalizacióncomo medio de comunicación y herramienta de marketing.

El marketing digital ofrece a las empresas una gran variedad de alternativas para favorecer la comercialización global y generar ingresos (Angeloni \& Rossi, 2021). Una de sus variantes más explotadas hoy en día es el marketing de influencia, basado en la recomendación (brand advocacy) realizada por terceros ajenos a la marca, ya sean influencers, empleados de la compañía, medios de comunicación o líderes de opinión (Suárez, 2021).

La eficacia de la influencia en las acciones de marketing ya fue confirmada antes de la popularidad de las redes sociales (López-Sáez \& Bustillos, 2008). Fue Daniel Edelman, en los años 60, quien acuñó el concepto al referirse a la influencia que los famosos y las celebridades ejercían sobre los consumidores, así como a la capacidad que tendrían estos de traducir opiniones en ventas (Castelló \& del Pino, 2015). El blog Influencity (2018), especializado en este tipo de marketing, recoge algunos casos de marketing de influencia que preceden al propio término, como es el caso de la marca Coca-Cola con Papá Noel, al que le cambiaron la imagen para dar soporte a su campaña en 1920, o la campaña que protagonizó Marilyn Monroe para la compañía de maquillaje Tru-Glo make up en 1953, si bien en estas campañas la influencia se ejercía por medio de líderes de opinión y no por influencers, en el significado actual de la palabra. Las estrategias sustentadas en influencers no llegan hasta los años 2009-2010, con el auge de los blogs y de MySpace, de modo que hoy en día recibimos la opinión de una persona que de alguna forma tiene cierto poder de influencia sobre nosotros (Santamaría \& Meana, 2017), pero que no es necesariamente conocida fuera de esa red social ni un líder de opinión consagrado 
en medios de comunicación tradicionales. Un estudio reciente concluye que los usuarios interactúan con personas influyentes porque les brindan contenido oportuno, entretenido e interesante (Bentley et al., 2021). Por lo tanto, sigue siendo necesario que el influencer sea una persona que cuente con credibilidad sobre un tema en específico y que, debido a su presencia e influencia en redes sociales, pueda convertirse en un prescriptor para una marca (Castelló \& del Pino, 2015). Esto exige que se trate de personas que tienen una gran cantidad de seguidores, pero a su vez esos seguidores cada vez valoran más la accesibilidad para poder interactuar con los influencers (Abidin, 2021). En el contexto español, Ibáñez-Sánchez y su equipo (2021) concluyeron que las colaboraciones marca-influencer de renombre, al ser comparadas con otras colaboraciones menos reconocidas, mejoran la actitud de los usuarios hacia el mensaje, sus intenciones de compra y la credibilidad percibida del influencer.

A pesar de que las ciudades y los lugares pueden actuar directamente como influencers (Banks, 2021), el uso de influencers en la promoción turística es una realidad (Gómez, 2018a). Según datos de IAB Spain (2020), la mayor asociación mundial de comunicación, publicidad y marketing digital, en España el 56\% de los usuarios de redes sociales siguen a influencers, de modo que se trata de las cuentas más seguidas después de las de otros usuarios de su entorno más cercano (96\%), Facebook, Instagram y YouTube son los sitios favoritos para seguirlos, aunque solo Instagram y YouTube experimentan un ascenso con respecto al año anterior ( $35 \%$ y $28 \%$, respectivamente), lo que justifica la necesidad de explorar el fenómeno en estas redes. Instagram es también la red social más utilizada cuando se trata de contratar a un influencer (70\% de los casos).

\section{INFLUENCERS COMO PRESCRIPTORES DE MARCAS}

El influencer surge de un concepto tan primario del marketing como lo es la identificación de necesidades, de modo que resuelve el interés que tenemos los consumidores de conocer la opinión y experiencia de otros usuarios que hayan tenido previamente relación con la marca. Al crearse una audiencia en forma de comunidad (Fernández, 2017), las empresas y las organizaciones han sabido reconocer una oportunidad de beneficio y una solución a la demanda de humanización de la marca en el entorno de las redes sociales.

Prescriptor es quien recomienda la compra o el consumo de un producto (Santiago, 2015), por lo general porque lo ha consumido antes, de manera tal que puede ocurrir que los usuarios acaben relacionando una marca con una persona determinada. Los brand avocates defienden la marca y la promocionan (Coobis, 2018), pero no reciben ingresos por ello, mientras que los influencers son generadores de opinión que la marca escoge expresamente para que comuniquen un mensaje específico y creen relaciones de identidad con el público objetivo. 
La selección de un influencer requiere de un estudio pormenorizado del perfil para dar respuesta a las aspiraciones de la marca. Se deben fijar unos objetivos clave y determinar el nivel de alcance más adecuado para la estrategia que se quiere implementar, acotar el público al que la empresa se quiere dirigir y planificar cómo quiere ser conocida la marca (Carrion, 2018; Santiago, 2015), pues son figuras que se mueven entre la intimidad y la publicidad, entre la autenticidad y la comercialización (Borchers, 2019). Entre las personas que ejercen algún tipo de influencia en el público, de acuerdo con la Pirámide del Influencer Engagement (Launchmetrics Content Team, 2015) podemos encontrar tres tipos:

a. Líderes de la comunidad y prosumers. Se trata de la categoría más numerosa, porque está formada por todos los consumidores actuales que se muestran comprometidos con una marca específica. Su influencia es menor, pero sus mensajes alcanzan gran credibilidad dentro de las propias comunidades.

b. Famosos o celebrities. Son fácilmente identificables debido a sus apariciones en los medios de comunicación tradicionales. Las colaboraciones que las marcas establecen suelen estar asociadas a contratos económicos.

c. Líderes de opinión, creadores de contenido o trend setters. Se trata de los influencers propiamente dichos, que crean y comparten contenido especializado con una comunidad.

Sin embargo, no todos los influencers son iguales, sino que es posible clasificarlos por la manera en la que adquirieron reconocimiento (Carrion, 2018):

a. Influencer de grandes masas. Su posicionamiento proviene del reconocimiento a su trabajo y de su estatus en los medios de comunicación tradicionales.

b. Influencer especializado. La influencia se genera por su conocimiento y comunicación, de modo que son una referencia para el público cuando tratan temas relacionados con su especialidad.

c. Micro influencer. Son personas destacadas en redes sociales que, aunque tengan menos seguidores que los anteriores, destacan por su gran engagement y por poseer una comunidad muy activa.

d. Influencer millenial. Son conocidos especialmente por los jóvenes pertenecientes a su generación.

e. Influencers de opinión. Aquellos que han conseguido notoriedad en redes sociales sin buscarlo activamente y cuyos contenidos son puramente personales. 
En otra clasificación más antigua (Santamaría \& Meana, 2017) se recogían diez categorías que hoy en día se aglutinan en la taxonomía anterior: (1) especialista de un sector, (2) especialistas de un producto, (3) influencer de un nicho, (4) influencer generalista, (5) influencer de tendencia, (6) influencer ocasional, (7) influencer de referencia, (8) influencer cautivo, (9) influencer por imitación, e (10) influencer anónimo. Con todo, la empatía, la profesionalidad, apostar por contenidos de calidad, la humildad, ser respetuoso, tener credibilidad y carisma, y ser reconocido y reconocible siguen siendo requisitos exigibles para cualquier influencer (López Herrera, 2018), pese a que lo verdaderamente importante es el engagement para generar compromiso entre marca y consumidores (Ure, 2018).

Ni los influencers son influyentes en todas las redes ni en todas las redes existen influencers, por lo que la empresa debe comenzar seleccionando la red en la que está presente su público objetivo y luego al influencer que le interesa de esa red en específico. Las redes sociales más utilizadas a la hora de explotar las estrategias del marketing de influencia son, por orden, Instagram y YouTube (Cabrera, 2019). Es evidente que todas las campañas se implementan con el propósito de captar nuevos clientes, fidelizar a los existentes o aumentar la comunidad de seguidores (BrandManic, 2018), por lo que cualquier acción enfocada a la influencia debe perseguir al menos uno de los siguientes objetivos (Navas, 2018):

1. Crear conciencia de marca y fortalecer la imagen. Para ello, se deben localizar personas de relevancia en las redes que marquen tendencia en el ámbito en cuestión y estén dispuestas a defender la marca. Existen herramientas para identificar personas influyentes (BuzzSumo, por ejemplo) y para recopilar y analizar contenido popular (Flocker, por ejemplo).

2. Incrementar el compromiso y las interacciones.

3. Mejorar las conversaciones. Las conversaciones suelen tener como fin último el comercial y, por lo tanto, los influencers pueden utilizarse de dos maneras: vinculando los productos a su contenido social o indicándoles que mencionen las redes sociales de la empresa contratante.

4. Conseguir datos de consumidores.

Este tipo de campañas suelen generar un buen retorno de la inversión (ROI), porque si el influencer tiene como audiencia el target de la marca y posee la capacidad de incentivar la venta del producto, es previsible que los ingresos sean mayores a la inversión realizada. Desde el punto de vista de los activos intangibles, se potencia el branding al conseguirse mayor visibilidad y lograr que se hable más de la marca (Pérez, 2016). Con todo, resulta fundamental que la organización seleccione al influencer adecuado para la promoción de su marca, pues algunos usuarios consideran que 
aquellos que emiten un exceso de publicidad acusan una pérdida de credibilidad en la transmisión del mensaje (Castelló \& del Pino, 2015), y también que la empresa sea transparente acerca de su relación publicitaria con el influencer (Peñalver, 2019).

Si los objetivos están bien planteados y la campaña se ejecuta con profesionalidad y solvencia, lo lógico es que los resultados del Earned Media Value (EMV) mejoren. El EMV es un método que se utiliza para calcular cuán importante es el contenido vinculado a las marcas a través de los esfuerzos de marketing (Dan, 2019) y, por lo tanto, proveniente de sus propios canales de comunicación con los públicos. Se calcula mediante una fórmula que mide el impacto de la estrategia de marketing digital, comparándolo con otros esfuerzos similares de marketing de alcance u outreach, de modo que es un buen referente para obtener una visión sobre la utilidad de los esfuerzos organizacionales en marketing digital.

El Informe Benchmark de Marketing de Influencers (Influencer Marketing Hub et al. (2019) indica que las empresas que usan bien el marketing de influencers obtienen grandes beneficios, incluso de hasta de $\$ 18$ por cada $\$ 1$ dólar invertido, aunque sitúan la media en $\$ 5,20$ de beneficio por cada dólar gastado. Los casos de empresas que pierden dinero son menos, el $25 \%$ de las encuestadas, y que, según explica el informe, se trata mayoritariamente de empresas que no comprenden el funcionamiento del marketing de influencia o que escogen a influencers cuyo mercado objetivo no es coincidente con el de la marca.

\section{Marketing de influencia en el sector turístico}

La web 2.0 ha cambiado la forma de viajar, tanto en el previaje (cuando se piensa el destino y en los desplazamientos) como durante el viaje y el posviaje (cuando se comparten las experiencias vividas) (Gómez, 2018b). Las recomendaciones de otros internautas generan en el usuario el deseo de experimentar por sí mismo e influyen de manera decisiva en sus decisiones. El deber del sector turístico, en su afán de adaptación a este cambio, es promover la creación de comunidades utilizando como plataforma las redes sociales, así como potenciarlas en sus estrategias de comunicación (Martínez-Sala et al., 2019), sobre todo considerando que 82\% de los usuarios de servicio de turismo siguen las redes sociales o el blog de influencers de viajes/moda o lifestyle (Axon Marketing y Communications, 2018). Lee y sus colaboradores (2021) han descubierto que son cuatro las razones que motivan que un usuario siga a influencers en Instagram: (1) autenticidad, (2) consumismo, (3) inspiración creativa y (4) envidia.

Los influencers de turismo o travel influencers se han convertido en figuras emblemáticas que han causado furor y han conseguido llegar a millones de personas (BrandManic, 2018). Se han transformado en los mejores guías de viajes, ya que al enseñar sus propias experiencias hacen partícipes a los seguidores, por lo que se 
han posicionado como una nueva figura de relevancia para las empresas de este sector (Basch, 2017). Hace unos años, se habría concebido como algo totalmente extravagante que un influenciador pretendiera hospedarse en un hotel a cambio de publicidad en redes sociales. Sin embargo, en la actualidad son las empresas las que hacen esfuerzos por localizarlos y concertar acciones, debido a que los resultados de este tipo de estrategias han superado cualquier expectativa (Blázquez, 2019). No obstante, los resultados de diferentes estudios muestran un enfoque unidireccional de la comunicación y, como consecuencia, una escasa explotación del potencial comunicativo de las redes sociales, lo que significa al mismo tiempo, el desatendimiento de las exigencias de los turistas 2.0 (Martínez-Sala et al., 2019).

A pesar de que los perfiles de los travel influencers son muy variados (Deloitte, 2020), 80\% de los usuarios considera que los influencers influyen en su decisión de manera relevante a la hora de escoger destinos turísticos (Axon Marketing y Communication, 2018). Se debe considerar que los travel users comparten experiencias; no hacen publicidad de manera directa, sino que presentan los servicios de manera más natural (Martínez-Sala et al., 2019), lo que provoca que el público perciba el contenido desde una perspectiva casi de amigo. Su contenido es de calidad y engancha, y por ello pueden conseguir un mayor engagement.

Si bien el discurso narrativo de los travel influencers se basa en compartir experiencias (Yllmaz et al., 2020), los impactos de los viajeros influyentes en sus seguidores se pueden clasificar en cuatro categorías: (1) efectos informativos, (2) efectos motivadores, (3) efectos como modelo a seguir, y (4) efectos comunitarios (Asan, 2021). Las prácticas de los influencers de viajes suelen atraer la atención de los seguidores, el autodescubrimiento, la participación de la comunidad y el intercambio de información (Gholamhosseinzadeh et al., 2021), de tal forma que actúan como una herramienta de marketing para orientar con éxito la toma de decisiones de destino de los turistas (Pop et al., 2021).

\section{OBJETIVOS Y METODOLOGÍA}

Este trabajo tiene como objetivo principal estudiar el uso que hacen de la figura de influencer en Instagram y YouTube las entidades turísticas que realizan una mayor inversión publicitaria en España para determinar si su uso aumenta el éxito de las publicaciones sociales. Asimismo, se establecen los siguientes objetivos secundarios:

- Analizar la posible relación existente entre una mayor inversión publicitaria y un mayor número de seguidores en redes sociales.

- Comprobar la relación entre el número de seguidores y la cantidad de likes que reciben las publicaciones de los influenciadores. 
- Detectar casos de marketing de influencia e identificar perfiles de influencers utilizados.

Se empleó una metodología de modelo mixto que combina técnicas descriptivas y analíticas. Se recurrió a una metodología cuantitativa descriptiva para explorar el fenómeno del marketing de influencia en YouTube e Instagram. La muestra de análisis la componen 10 empresas, tanto públicas como privadas, relacionadas con el sector turístico (tabla 1), que acorde con los datos publicados por InfoAdex en 2019, realizaron más inversión publicitaria en España ese año. De igual modo, fue necesario identificar las 10 cuentas de influencers españoles especializados en turismo con más seguidores tanto en Instagram como en YouTube (tablas 2 y 3 ) para comprobar de qué forma podrían haber colaborado con esas entidades.

La investigación se realizó en tres fases. La primera consistió en examinar las publicaciones permanentes que se habían subido a Instagram y YouTube en los meses de enero y febrero de 2020 en las cuentas de las empresas. En ambas redes sociales se consideraron cuatro patrones comunes: (1) fecha de publicación, (2) número de me gusta, (3) número de comentarios y (4) menciones a influencers. En el caso de YouTube también se consideró el número de visualizaciones de cada video (5). El estudio de estos ítems permite conocer el engagement generado y las rutinas de publicación de cada organización, así como la manera en la que se utiliza el marketing de influencia y los perfiles de influencers escogidos por las entidades. En la segunda fase, del 27 de abril al 3 de mayo de 2020, se analizó el uso del contenido autodestruible o efímero, específicamente las historias de Instagram en los perfiles de las entidades seleccionadas, conforme a los siguientes ítems: (1) publicación, (2) fecha, (3) hora aproximada, (4) swipe up e (5) influencers etiquetados. Se realizó, por lo tanto, un seguimiento de la cantidad de historias que se subían con el propósito de determinar rutinas, horas de publicación, así como el posible empleo de influencers y swipe up en el storytelling. En una última fase, se analizó el Earned Media Value (Valor mediático ganado) a través de la herramienta Influence4You, que utiliza como base los valores de Ayzenberg, es decir, un modelo de conversión en valor monetario de las principales KPI de redes sociales y que son considerados los estandarizados para este tipo de medida.

Los datos se procesaron en fichas de análisis diseñadas ad hoc y se expresan en términos porcentuales.

\section{RESULTADOS}

En la tabla 1 se recogen las diez empresas españolas relacionadas con el turismo que cumplieron con el criterio de inclusión de realizar una mayor inversión publicitaria en 2019 (todas superan los tres millones de euros). 


\begin{tabular}{|c|c|c|c|c|c|c|}
\hline $\mathrm{N}^{\circ}$ & Entidad & $\begin{array}{l}\text { Inversión } \\
\text { publicitaria } \\
\text { en el } 2019 \\
\text { (en euros) }\end{array}$ & $\begin{array}{c}\text { Nombre } \\
\text { de usuario en } \\
\text { YouTube }\end{array}$ & $\begin{array}{c}\text { Suscriptores } \\
\text { en YouTube } \\
\text { ¿en miles? }\end{array}$ & $\begin{array}{c}\text { Nombre } \\
\text { de usuario } \\
\text { en Instagram }\end{array}$ & $\begin{array}{l}\text { Seguidores } \\
\text { en Instagram }\end{array}$ \\
\hline 1 & $\begin{array}{c}\text { El Corte } \\
\text { Inglés Viajes }\end{array}$ & 27.399 .056 & viajeselcorteingles & 8490 & @viajeselcorteingles & 74.700 \\
\hline 2 & $\operatorname{Renfe}^{* *}$ & 6.599 .785 & renfe & 10.900 & @renfe & 44.900 \\
\hline 3 & Iberia & 5.800 .004 & iberia & 32.200 & @iberia & 394.000 \\
\hline 4 & $\begin{array}{l}\text { Nautalia } \\
\text { Viajes }\end{array}$ & 4.923 .445 & Nautalia Viajes & 1310 & @nautaliaviajes & 7857 \\
\hline 5 & $\begin{array}{c}\text { MSC } \\
\text { Cruceros }\end{array}$ & 4.087 .588 & $\begin{array}{c}\text { MSC Cruceros } \\
\text { España }\end{array}$ & 7.710 & @msccruisesofficial & 576.000 \\
\hline 6 & $\begin{array}{l}\text { Costa } \\
\text { Cruceros }\end{array}$ & 4.004 .010 & Costa Cruceros & 3870 & @costacruisesofficial & 168.000 \\
\hline 7 & Booking.com & 3.940 .789 & Booking.com & 38.700 & @bookingcom & 1.300 .000 \\
\hline 8 & $\begin{array}{l}\text { Grimaldi } \\
\text { Group }\end{array}$ & 3.564 .808 & Grimaldi Lines & * & @grimaldilines & 9725 \\
\hline 9 & $\begin{array}{c}\text { Generalitat } \\
\text { Valenciana** }\end{array}$ & 3.383 .662 & $\begin{array}{l}\text { Generalitat } \\
\text { Valenciana }\end{array}$ & 4870 & @generalitatvalenciana & 34.500 \\
\hline 10 & $\begin{array}{l}\text { Avoris Retail } \\
\text { Division }\end{array}$ & 3.270 .860 & $B$ the travel brand & 866 & @bthetravelbrand & 53.000 \\
\hline
\end{tabular}

* Información no disponible

** Entidades públicas

Tabla 1. Lista de empresas turísticas con mayor inversión publicitaria en España y presencia en YouTube e Instagram

Fuente: Elaboración propia, con datos de InfoAdex (2019).

Se incluyen sus nombres de usuario en Instagram y en YouTube, así como el número de seguidores en cada una de esas redes sociales. Se observa una preferencia clara de los usuarios por Instagram, ya que todas las empresas tienen más seguidores en esa red que en YouTube. También se puede comprobar en dicha tabla que las entidades que más dinero invirtieron en publicidad en 2019 no coinciden con aquellas que cuentan con más seguidores en las redes sociales.

No obstante, el número de seguidores de una cuenta en Instagram no implica necesariamente una mayor interacción social (no se tiene en cuenta YouTube, porque en esa red el número de likes no implica diálogo social). En efecto, la relación entre número de seguidores y likes es mayor en aquellas empresas que tienen menos seguidores (gráfico 1), es decir, porcentualmente se constatan menos seguidores que den me gusta a las publicaciones en aquellas cuentas que tienen más followers. Así, Renfe (empresa de ferrocarriles) es la entidad en la que los usuarios dan más like a las publicaciones $(37,48 \%)$ y, sin embargo, se sitúa como la cuarta con menos seguidores. 


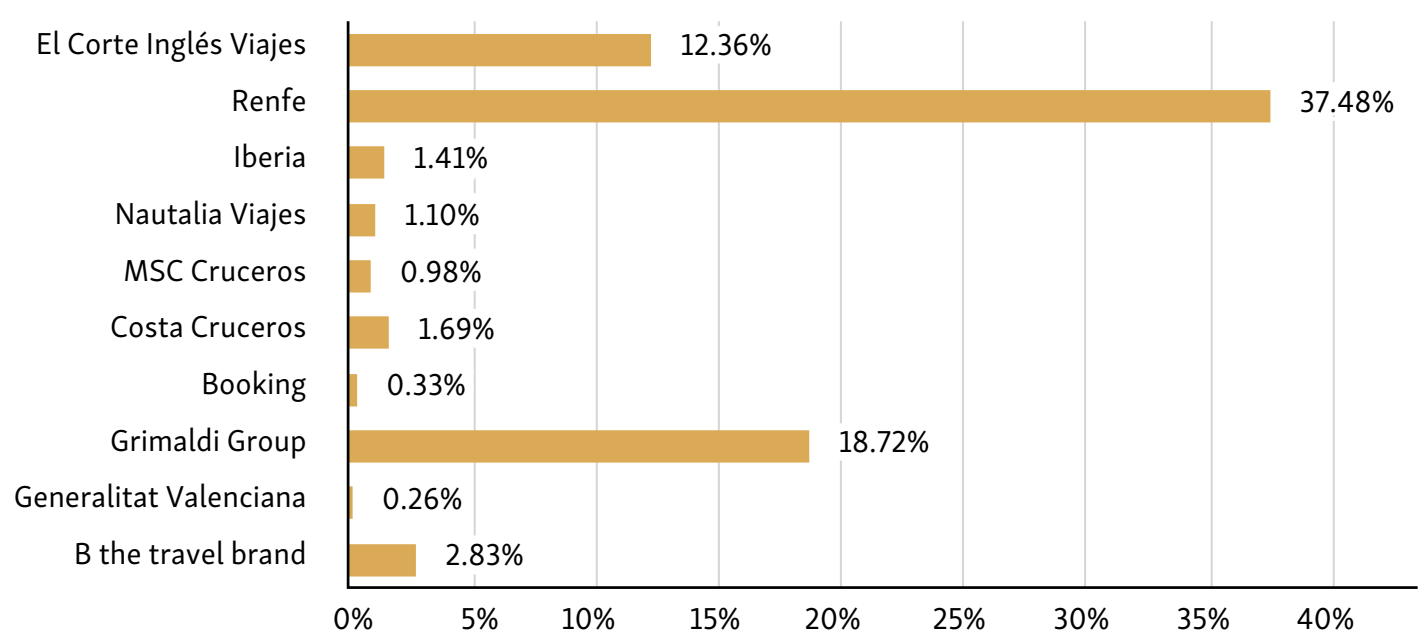

Gráfico 1. Relación entre seguidores y media de me gusta en Instagram

Fuente: Elaboración propia.

Con todo, se aprecia un aumento en la media de me gusta en aquellas publicaciones de las empresas estudiadas en que se etiquetan influencers (gráfico 2). El 60\% de las entidades recurre a influencers para sus publicaciones y se observa que las publicaciones en las que están etiquetadas estas personas son las que mayor éxito tienen, lo que evidencia la importancia de este tipo de prácticas en la comunicación digital de las entidades.

En cuanto a los perfiles de influencers (Carrion, 2018) a los que recurren las empresas (gráfico 3), se aprecia predisposición por los influencers especializados (sobre todo travel influencers con seguidores especialmente interesados en viajes y turismo), y, a continuación, por los influencers de grandes masas (futbolistas, personas famosas, actrices...). Menos utilizados son los perfiles de aquellos influencers que tienen menos seguidores pero un engagement elevado (micro influencers) y aquellos cuya fama se debe a que suelen compartir su día a día (influencers de opinión).

De entre las 10 personas españolas especializadas en viajes más seguidas en Instagram (tabla 2) o YouTube (tabla 3), solo dos fueron mencionadas por alguna empresa. Se trata de las cuentas de @molaviajar y Paco Nadal, que colaboraron con MSC Cruceros y B the travel brand respectivamente. Se diagnostica, pues, una preferencia por cuentas especializadas frente a las que acumulan un mayor número de seguidores, y también por micro influencers con un nicho de mercado más reducido pero mayor arraigo entre la comunidad social. La especialización temática de las cuentas y nivel alto de engagement resultan los dos factores determinantes que tienen en cuenta las empresas a la hora de seleccionar influencers para sus campañas de marketing digital. 
Media de 'me gusta' que obtiene una publicación sin mención a influencer (en miles)

- Media de 'me gusta' que obtiene una publicación con mención a influencer (en miles)

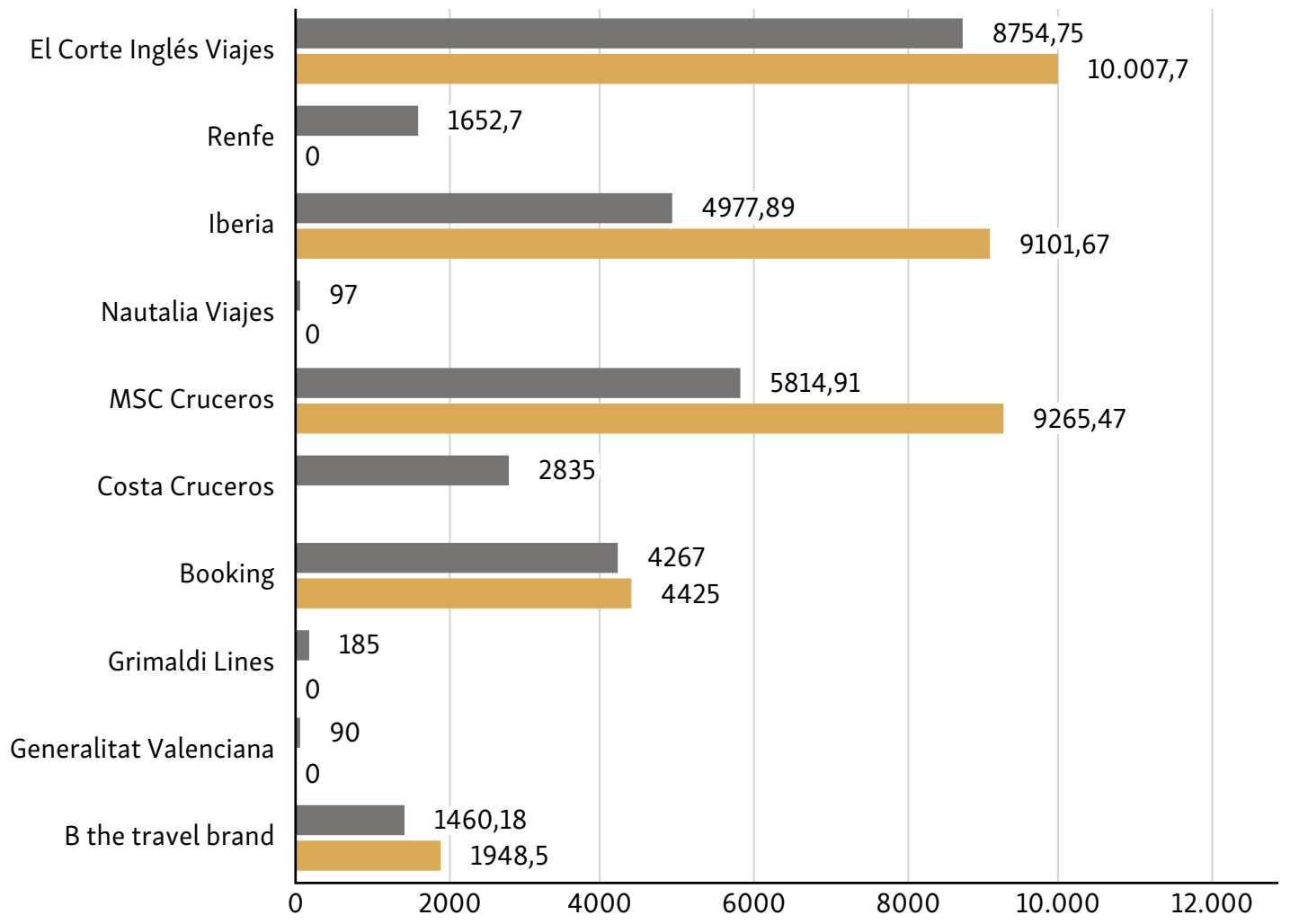

\section{Gráfico 2. Media de me gustan likes en publicaciones de Instagram de empresas con y sin mención influencers}

Fuente: Elaboración propia.

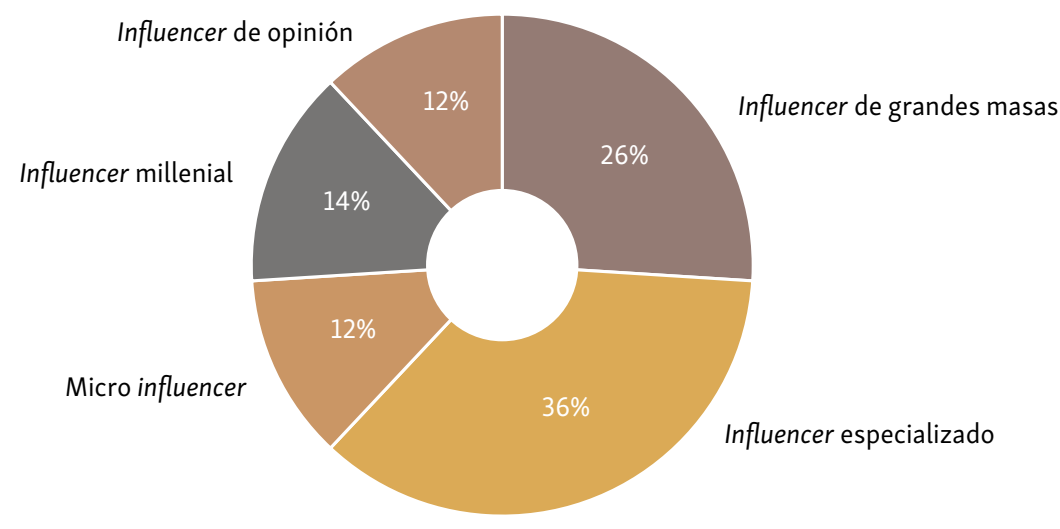

Gráfico 3. Tipos de influencers utilizados en Instagram y YouTube por las empresas de turismo españolas

Fuente: Elaboración propia. 


\begin{tabular}{|c|c|c|c|}
\hline Número & Nombre completo & Usuario & $\begin{array}{l}\text { Número de } \\
\text { seguidores }\end{array}$ \\
\hline \multicolumn{4}{|c|}{ Instagram } \\
\hline 1 & Nicanor García & @nicanorgarcia & 745.000 \\
\hline 2 & Elena Ortega & @misswinter & 491.000 \\
\hline 3 & Oliver Vegas & @ovunno & 421.000 \\
\hline 4 & Marina Comes & @marinacomes & 303.000 \\
\hline 5 & Kike Arnaiz & @kikearnaiz & 233.000 \\
\hline 6 & Roger Carles y Vanessa Santiago & @viajeroscallejeros & 181.000 \\
\hline 7 & Enrique Álex Garde & @enriquealex & 151.000 \\
\hline 8 & Benjamin Thorpe & @benjithorpe & 139.000 \\
\hline 9 & $\begin{array}{l}\text { Adrián Rodríguez, Gosia Bendrat, } \\
\text { Daniela y Oliver Rodríguez }\end{array}$ & @molaviajar & 128.000 \\
\hline 10 & Gabriel Samper & @kainxs & 102.000 \\
\hline \multicolumn{4}{|c|}{ YouTube } \\
\hline 1 & Pau Clavero & Clavero & 957.000 \\
\hline 2 & Enrique Álex Garde & Enrique Álex & 414.000 \\
\hline 3 & Carlos García & Charly Sinewan & 406.000 \\
\hline 4 & $\begin{array}{c}\text { Adrián Rodríguez, Gosia Bendrat; Daniela } \\
\text { y Oliver Rodríguez }\end{array}$ & Molaviajar & 315.000 \\
\hline 5 & Izhan Galiana & Izhan & 307.000 \\
\hline 6 & Agustín Ostos & Soy Tribu & 136.000 \\
\hline 7 & Judith Tiral & Judith Tiral & 104.000 \\
\hline 8 & Chistian Corom & Christian Corom & 85.200 \\
\hline 9 & Rubén Alonso & Ruben y El Mundo & 70.300 \\
\hline 10 & Paco Nadal & Paco Nadal & 45.800 \\
\hline
\end{tabular}

Tabla 2. Lista de los 10 influencers españoles dedicados a viajes con más seguidores en Instagram y YouTube

Fuente: Elaboración propia con datos de Martín (2019) y Ruiz (2020).

En la tabla 3 se recogen todos los influencers utilizados por las distintas entidades tanto en publicaciones efímeras o de contenido autodestruible (historias de Instagram) como permanente. Se puede comprobar que, salvo dos, los influencers que figuran en la relación no se corresponden con los que se localizan entre los 10 primeros de los dedicados a viajes con más seguidores en Instagram y YouTube (tabla 2), sino con perfiles que pertenecen a la categoría adulto-joven, dependiendo del target de la marca, con seguidores de ámbito internacional y conocidos por viajar mucho, de modo que todos mantienen una relación estrecha con el turismo y la implementación de estrategias de marketing de viajes. 


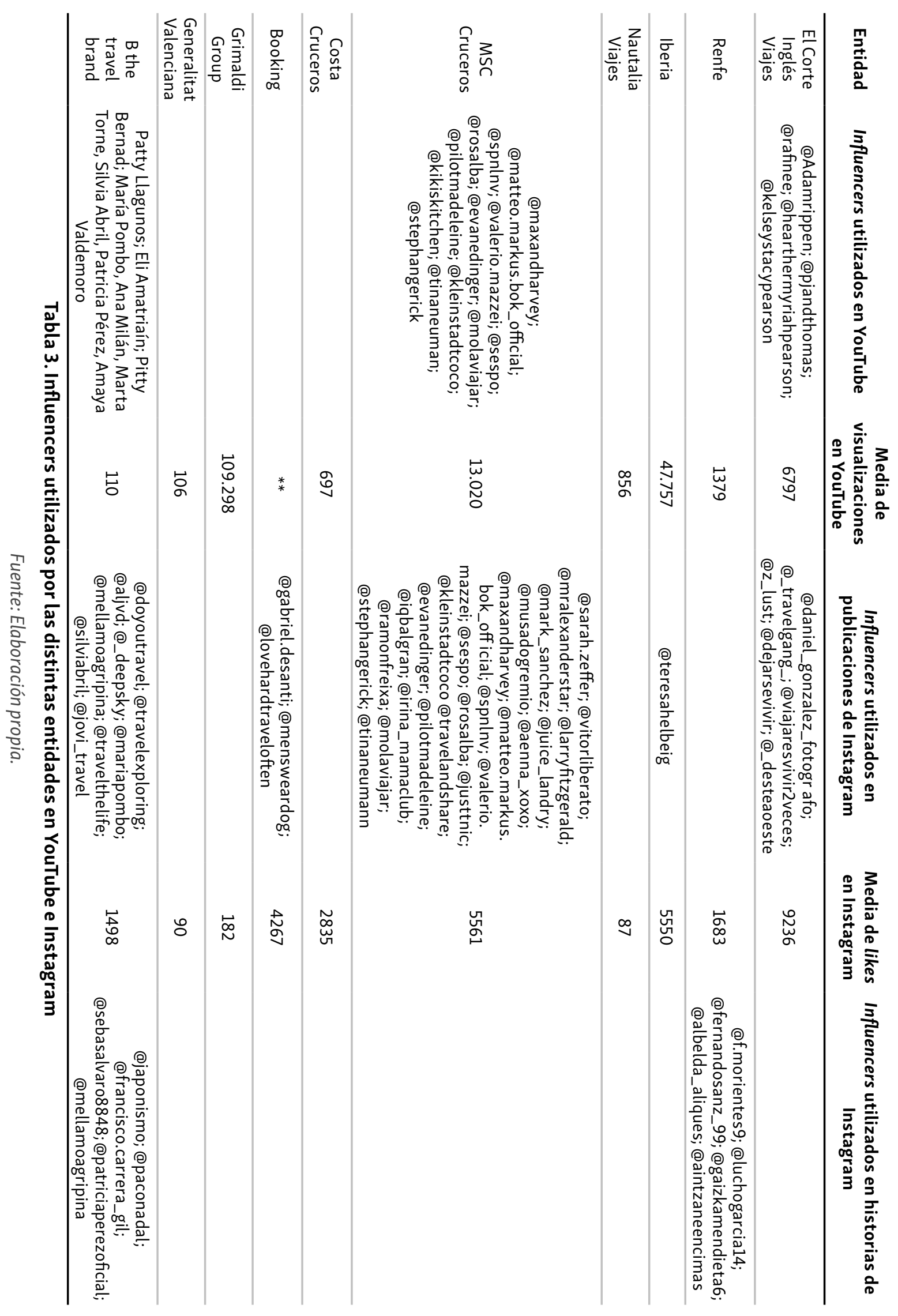




\begin{tabular}{|c|c|c|c|c|c|c|c|}
\hline Entidad & $\begin{array}{l}\text { Publicaciones } \\
\text { en YouTube }\end{array}$ & $\begin{array}{l}\text { Influencers } \\
\text { etiquetados } \\
\text { en YouTube }\end{array}$ & $\begin{array}{l}\text { Publicaciones } \\
\text { en Instagram }\end{array}$ & $\begin{array}{c}\text { Influencers } \\
\text { etiquetados } \\
\text { en Instagram }\end{array}$ & $\begin{array}{l}\text { Historias } \\
\text { subidas }\end{array}$ & $\begin{array}{c}\text { Influencers } \\
\text { etiquetados } \\
\text { en las } \\
\text { historias }\end{array}$ & $\begin{array}{c}\text { Uso } \\
\text { total de } \\
\text { influencers }\end{array}$ \\
\hline $\begin{array}{l}\text { El Corte } \\
\text { Inglés } \\
\text { Viajes }\end{array}$ & 8 & $5(41,66 \%)$ & 26 & $7(58,33 \%)$ & 1 & 0 & 12 \\
\hline Renfe & 2 & 0 & 23 & 0 & 29 & $6(100 \%)$ & 6 \\
\hline Iberia & 10 & 0 & 21 & $1(100 \%)$ & 0 & 0 & 1 \\
\hline $\begin{array}{l}\text { Nautalia } \\
\text { Viajes }\end{array}$ & 3 & 0 & 16 & 0 & 4 & 0 & 0 \\
\hline $\begin{array}{c}\text { MSC } \\
\text { Cruceros }\end{array}$ & 24 & $13(32,50 \%)$ & 71 & $27(67,50 \%)$ & 44 & 0 & 40 \\
\hline $\begin{array}{c}\text { Costa } \\
\text { Cruceros }\end{array}$ & 1 & 0 & 16 & 0 & 12 & 0 & 0 \\
\hline Booking & 0 & 0 & 9 & $\begin{array}{c}3 \\
(100 \%)\end{array}$ & 0 & 0 & 3 \\
\hline $\begin{array}{l}\text { Grimaldi } \\
\text { Group }\end{array}$ & 9 & 0 & 17 & 0 & 4 & 0 & 0 \\
\hline $\begin{array}{l}\text { Generalitat } \\
\text { Valenciana }\end{array}$ & 34 & 0 & 118 & 0 & 3 & 0 & 0 \\
\hline $\begin{array}{c}\text { B the travel } \\
\text { brand }\end{array}$ & 10 & $8(34,78 \%)$ & 64 & $9(39,13 \%)$ & 47 & $6(26,08 \%)$ & 23 \\
\hline MEDIA & 10,1 & 2,6 & 38,1 & 4,7 & 14,4 & 1,2 & \\
\hline
\end{tabular}

Tabla 4. Número de publicaciones, historias e influencers etiquetados en Instagram y YouTube Fuente: Elaboración propia.

No se evidencia una estrategia comunicativa coincidente entre las distintas entidades que permita determinar o establecer unos parámetros conductuales comunes, aunque sí una preferencia por publicar más y por utilizar más influencers en Instagram que en YouTube (tabla 4). De igual modo, en el contenido autodestruible se registra un uso menor de la influencia que en las publicaciones permanentes.

Tras calcular el Earned Media Value (gráfico 4), es decir, el método para rastrear cualquier contenido o comunicación relacionada con la marca, se evidenció que el uso del marketing de influencia permite aumentar el valor de las redes sociales de las empresas, porque aumenta el número de interacciones sociales y mejora las KPI establecidas según los valores de Ayzenberg determinados por Influence4You. Aunque hay casos como Booking, en los que este cambio es casi imperceptible, hay otros como MSC Cruceros en donde la diferencia entre utilizar o no influencers supone una gran mejora del valor. Si habíamos dicho que un mayor número de seguidores no implica mayor interacción social, ahora se puede determinar que un número alto de seguidores es un factor determinante para otorgar más valor a la cuenta, ya que los perfiles con más seguidores también obtienen mejores resultados de Earned Media Value. 


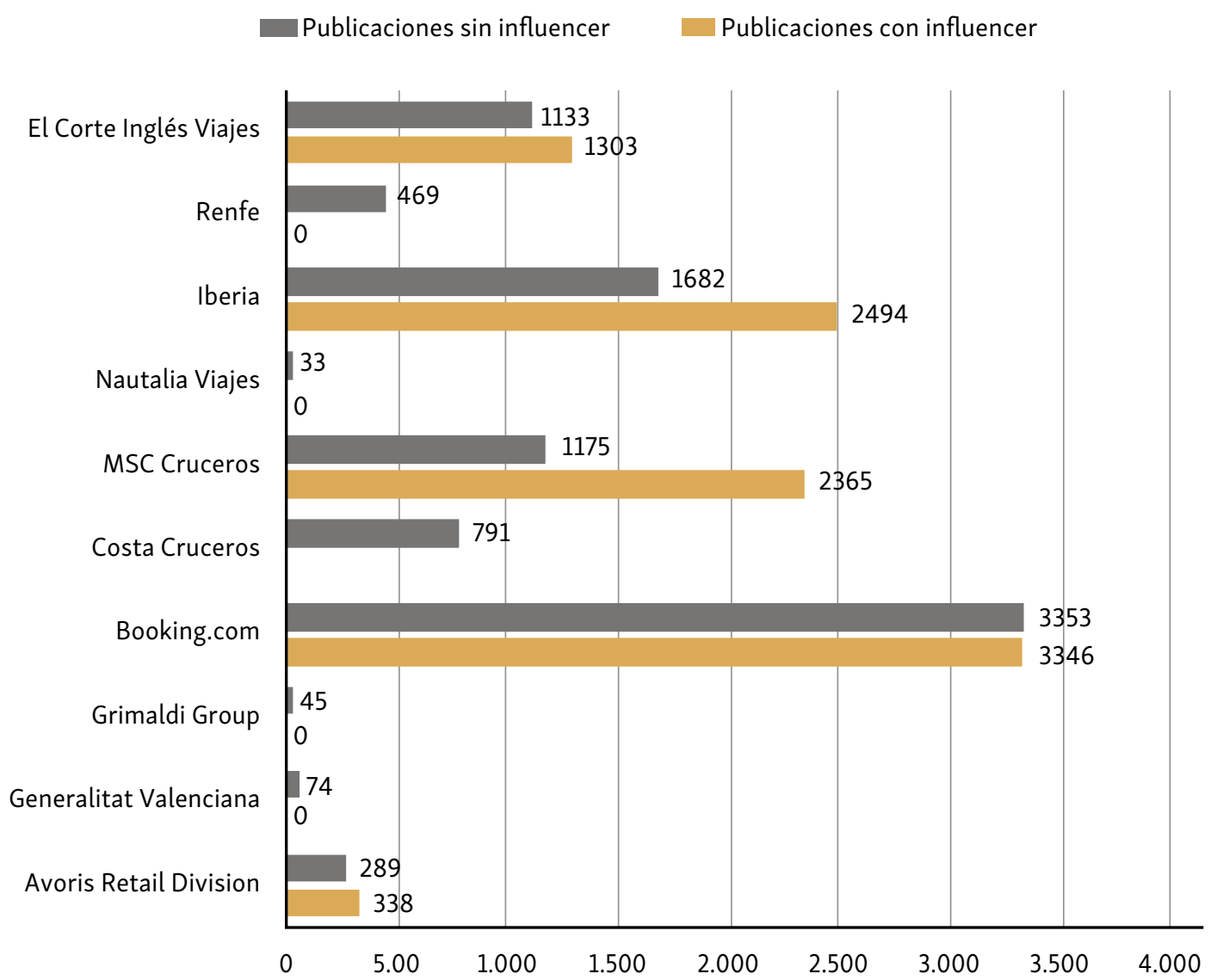

\section{Gráfico 4. Earned Media Value de las entidades turísticas en España}

Fuente: Elaboración propia.

\section{DISCUSIÓN Y CONCLUSIONES}

La inversión publicitaria de las entidades turísticas españolas no está destinada en su totalidad a las plataformas en línea, por lo que no existe relación directa entre una mayor inversión en publicidad y un mayor éxito en redes sociales o un mayor ejercicio del marketing de influencia. De hecho, a la vista de los resultados obtenidos en la investigación, tampoco puede establecerse una relación indirecta, pues todo hace pensar que parte de la inversión publicitaria se destina a medios tradicionales, ya que no existe coincidencia entre las empresas que más invierten en publicidad y las que tienen más seguidores en redes, aunque sí se descubre que las publicaciones con menciones a influencers obtienen significativamente más likes que las que no los utilizan.

De todas formas, en el sector turístico no todas las redes sociales despiertan el mismo interés entre los usuarios. Los resultados coinciden con las tendencias detectadas por IAB Spain (2020), al confirmarse mayor predilección por Instagram que por YouTube, algo que puede explicarse porque cuando se participa en una red de videos como YouTube se suele buscar contenido específico, mientras que 
Instagram se emplea para visualizar contenido que suben las cuentas que sigue el usuario y que le interesan. Es por eso por lo que siempre que el contenido de una cuenta de Instagram resulta relevante el usuario acostumbra a seguirla para asegurarse de estar enterado de todas las novedades, algo que no sucede en YouTube con tanta frecuencia.

Con todo, el éxito de una organización en redes sociales no solo se basa en tener un gran número de seguidores, sino en el compromiso que estos demuestran hacia la marca, esto es, en el engagement. El número de likes o la cantidad de veces que se publica contenido son factores que determinan el engagement de una cuenta, pero no se evidencia que una cantidad elevada de seguidores se transforme necesariamente en un número elevado de likes en cada publicación, sino que los datos demuestran que las cuentas más seguidas no coinciden con las que más me gusta reciben por publicación. Así, por ejemplo, Booking.com es la cuenta más seguida tanto en YouTube $(38,7 \mathrm{~K})$ como en Instagram (1,3 M), pero queda en penúltima posición en cuanto a media de likes por publicación, pues solo 0,33\% de sus seguidores da me gusta a sus publicaciones. Es importante que la comunidad científica tenga en cuenta que un mayor número de seguidores no siempre equivale a una mayor interacción social, sino que el valor de las cuentas en redes sociales depende más del engagement de la comunidad que de las cifras de seguimiento. De ahí el interés por los micro influencers que no tienen un gran número de seguidores, pero cuya comunicación es altamente efectiva. Al observar la relación entre seguidores y me gusta (porcentaje de seguidores que da like a las publicaciones) se comprobó que es en las entidades con menor número de seguidores donde estos dan más me gusta al contenido subido. Específicamente sobresale el caso de Renfe, donde 37,48\% de los seguidores da like a pesar de situarse como la cuarta entidad con menos followers del conjunto estudiado. En consecuencia, no se constata relación directa entre éxito en redes sociales y número de seguidores, sino que el éxito depende del engagement y este, a su vez, de la interacción que los usuarios practiquen con la marca, que en el ámbito turístico es mayor en aquellas comunidades más reducidas. Sin embargo, sí se constata que el uso de influencersen las publicaciones aumenta la interacción social (número de likes) y el Earned Media Value y, por lo tanto, el return on investiment (ROI), de modo que una mayor inversión en contratación de estrategias de marketing de influencers repercute de forma directa en la cuenta de resultados de la organización.

Los resultados evidencian que seis de las 10 de las entidades estudiadas hacen uso del marketing de influencia. Los perfiles profesionales utilizados varían mucho dependiendo de la empresa y de la estrategia comunicativa que se esté efectuando en cada momento. En el caso de El Corte Inglés Viajes, por ejemplo, 50\% de los influencers utilizados eran especialistas en un solo sector -el turismo-, mientras 
que Renfe utilizó únicamente figuras conocidas para elaborar historias como parte de un challenge y todos ellos, al ser personalidades muy conocidas del mundo del fútbol, se consideran influencers de grandes masas (Carrion, 2018).

El perfil más utilizado en las empresas turísticas españolas es el influencer especializado, seguido del influencer de grandes masas. En el primer caso, las áreas de especialización de influencia son el turismo y los viajes (travel influencers), mientras que aquellas celebridades de grandes masas son deportistas de élite y actrices/actores. Queda demostrada la preferencia de las empresas por este tipo de perfiles frente a los influencers más relevantes (con mayor número de seguidores) tanto en YouTube como en Instagram, y también su inclusión en las publicaciones permanentes frente a las efímeras o autodestruibles. Si bien el uso de micro influencers en la comunicación social del turismo en España se reduce a 12\%, al observar los datos en conjunto se diagnostica preferencia por este tipo de perfiles y por cuentas especializadas (38\% en total) frente a influencers de grandes masas (36\%). Los resultados coinciden, pues, con estudios previos que demuestran que en la comunicación de viajes los usuarios apuestan por quienes comparten experiencias (Yllmaz et al., 2020) o se muestran cercanos a la comunidad (Abidin, 2021) a la hora de seleccionar los perfiles que les resultan interesantes (Bentley et al., 2021).

Cuando se comparó el éxito de las publicaciones que contenían menciones a influencers y aquellas que no las tenían, se observó que obtienen mayor número de likes aquellas en las que se utiliza esa herramienta tanto en comparativa con otras publicaciones de la misma empresa como al comparar las distintas entidades. Se concluye, por lo tanto, que la utilización del marketing de influencia es útil para aumentar el número de me gusta de las publicaciones, ya que los usuarios sienten mayor interés al percibir este tipo de colaboraciones. Esto incita al like, que incrementa el engagement, el Earned Media Value y, en definitiva, garantiza un mejor retorno económico de la inversión. Estos datos van en la línea de los expuestos en el Informe Benchmark de Marketing de Influencers (Influencer Marketing Hub et al., 2019), que afirma que el uso de influencers proporciona beneficios a las empresas. De igual modo, al analizar el Earned Media Value también se comprobó que cuando las entidades turísticas emplean marketing de influencia aumenta el valor de sus redes sociales.

La empresa que utiliza más influencers en YouTube es MSC Cruceros. Esta compañía también destaca en Instagram por su forma de comunicar a través de las publicaciones autodestruibles, que consiguen gran feedback gracias a iniciativas como encuestas y cuestionarios que implican la participación de los públicos. Otra empresa que sobresale en Instagram por el uso de influencers es B the travel brand, donde continuamente aparecen personajes públicos de relevancia, aunque sin 
circunscribirse a un perfil específico, de modo que pueden aparecer actrices como instagramers o deportistas.

Finalmente, al comparar la comunicación empleada en YouTube y en Instagram, se llega a la conclusión de que en la primera red social se busca menos interactividad, es decir, las entidades no pretenden un diálogo con la comunidad, sino que simplemente suben contenido y esperan que este sea consumido. En contraposición, en Instagram la comunicación presenta mayor interactividad y engagement debido, sobre todo, al uso de las historias. Instagram es, además, la red social más elegida por las entidades para subir contenido creado por influencers.

Como futuras líneas de investigación se propone estudiar el fenómeno de los influencers en otros sectores para poder establecer una comparación con los resultados obtenidos en este caso. La limitación de la muestra de este trabajo, establecida por una inversión publicitaria igual o superior a los tres millones de euros, podría subsanarse ampliando la muestra a otras empresas con un menor volumen de inversión, incluso para determinar si existen diferencias sustanciales en función de ese dato. Otra de las limitaciones de la investigación ha sido la imposibilidad de concretar qué porcentaje de la inversión publicitaria se destina a estrategias digitales y, dentro de ella, a redes sociales y, a su vez, al marketing de influencia. En todo caso, cabe destacar la relevancia de este estudio para la comunidad científica y para investigaciones futuras, especialmente por los resultados relativos a que el empleo de influencers mejora el engagement, el valor $\mathrm{y}$, en consecuencia, el ROI.

\section{REFERENCIAS}

Abidin, C. (2021). Singaporean Influencers and Covid-19 on Instagram Stories. Celebrity Studies, 12(4), 693-698. https://doi.org/10.1080/19392397.2021.1967604

Angeloni, S. \& Rossi, C. (2021). An analytical model for comparing the profitability of competing online marketing channels: Search engine marketing versus e-commerce marketplace. Journal of Marketing Theory and Practice, 29(4), 534-549. https://doi.org/10.1080/10696679.2021.1879656

Asan, K. (2021). Measuring the impacts of travel influencers on bicycle travellers. Current Issues in Tourism. https://doi.org/10.1080/13683500.2021.1914004

Axon Marketing y Communication (2018). Tendencias del marketing de influencers en el sector turismo para el 2018 (Influencer Marketing Trends in the Tourism Sector for 2018). https://cip.org.ar/wp-content/uploads/2018/06/Influencers-y-Turismo-2018.pdf

Banks, D. (2021). The attention economy of authentic cities: How cities behave like influencers. European Planning Studies. https://doi.org/10.1080/09654313.2021.1882947

Basch, M. (2017, December 6). Influencers viajeros: a quién seguir si te vas de vacaciones (Traveling influencers: who to follow if you go on vacation). La Nación. https://www.lanacion. com.ar/lifestyle/influencers-viajeros-a-quien-seguir-si-te-vas-de-vacaciones-nid2088610/ 
Bentley, K., Chu, C., Nistor, C., Pehlivan, E., \& Yalcin, T. (2021). Social media engagement for global influencers. Journal of Global Marketing, 34(3), 205-219.

https://doi.org/10.1080/08911762.2021.1895403

Blázquez, A. (2017).Análisis de la promoción del destinoturístico “Cartagena" a través de las redes sociales (Analysis of the promotion of the tourist destination "Cartagena" through social networks) (Undergraduate thesis, Universidad Politécnica de Cartagena). https://repositorio.upct.es/ bitstream/handle/10317/6022/tfg-bla-ana.pdf?sequence=lyisAllowed=y

Borchers, N. (2019). Social Media Influencers in Strategic Communication. International Journal of Strategic Communication, 13(4), 255-260. https://doi.org/10.1080/1553118X.2019.1634075

BrandManic. (2018). Estudio sobre Marketing de Influencers en España, 2018 (Study on Marketing of Influencers in Spain, 2018). https://www.brandmanic.com/estudio-marketing-deinfluencers-en-espana-2018/

Cabrera, M. (2019). Marketing de Influencia en Instagram (Influencer Marketing on Instagram) (Undergraduate thesis, Universidad de Sevilla). https://idus.us.es/handle/11441/65916?

Carrion, L. (2018, April 26). Guía para elegir al influencer perfecto para tu marca (Guide to choosing the perfect influencer for your Brand). MailRelay. https://blog.mailrelay.com/es/2018/04/26/elegir-influencer

Castelló, A. \& del Pino, C. (2015). La comunicación publicitaria con influencers (Advertising communication with influencers). Revista Digital De Marketing Aplicado, 1(14), 40-50. https://doi.org/10.17979/redma.2015.01.014.4880

Coobis. (2018, August 16). Brand advocate: qué es, sus beneficios y como desarrollar tu estrategia (Brand advocate: what it is, its benefits and how to develop your strategy). Coobis. https://coobis.com/es/cooblog/brand-advocate/

Dan (2019, July 18). How valuable is the online media value obtained from share by share? TalkWalker. https://www.talkwalker.com/es/blog/earned-media-value

Deloitte. (2020). Influencers: ¿Cómo tenerlos en cuenta? (Influencers: How to take them into account?). Deloitte. https://www2.deloitte.com/es/es/pages/operations/articles/fiturinfluencers-redes-sociales.html

ESIC (2018, March). 12 nuevos tipos de Marketing Digital. (12 New types of Digital Marketing). ESIC Business \& Marketing school. https://www.esic.edu/rethink/ marketing-y-comunicacion/12-nuevos-tipos-marketing-digital

Fernández, A. (2017). Estudio del origen de la figura del influencia $y$ análisis de su poder de influencia en base a sus comunidades (Study of the origin of the figure of influence and analysis of their power of influence based on their communities) (Undergraduate thesis, Universidad Pompeu Fabra). https://repositori.upf.edu/bitstream/handle/10230/36313/ Fernandez_2017.pdf? sequence $=1$ yisAllowed $=y$

Galindo, E. (2018, August 3). ¿Qué es el Inbound marketing? (What is inbound marketing?). Increnta. http://increnta.com/es/blog/que-es-el-inbound-marketing/

Gholamhosseinzadeh, M., Chapuis, J., \& Lehu, J. (2021). Tourism netnography: How travel bloggers influence destination image. Tourism Recreation Research. https://doi.org/10.1080/02508281.2021.1911274 
Gómez, A. (2018a). Influencers para la promoción de destinos turísticos (Influencers for the promotion of tourist destinations). Marketing Visionario, 6(2), 41-61. http://ojs.urbe.edu/index.php/market/article/view/1661

Gómez, B. (2018b). El influencer: herramienta clave en el contexto digital de la publicidad engañosa (The influencer: a key tool in the digital context of false advertising). methados. Revista de Ciencias Sociales, 6(1), 151-153. ttps://doi.org/10.17502/m.rcs.v6i1.212

IAB Spain. (2020). Estudio de Redes Sociales 2020 (Social networks study). IAB Spain. https://iabspain.es/estudio/estudio-redes-sociales-2020/

Ibáñez-Sánchez, S., Flavián, M., Casaló, L., \& Belanche, D. (2021). Influencers and brands successful collaborations: A mutual reinforcement to promote products and services on social media. Journal of Marketing Communications. https://doi.org/10.1080/13527266.2021.1929410

Influencer Marketing Hub, NeoReach \& Viral Nation (2019). Informe Benchmark de Marketing de Influencers: 2019 (Influencers Marketing Benchmark Report: 2019). https://influencermarketinghub.com/resources/influencer-marketing-benchmarkreport-2019/

Influencity. (2018, December 11). Antecedentes del marketing de influencia (Influencer Marketing Background). Influencity Blog. https://influencity.com/blog/es/antecedentesdel-marketing-de-influencia/

Infoadex. (2019). Estudio InfoAdex de la Inversión Publicitaria en España 2019 (InfoAdex Study of Advertising Investment in Spain 2019) https://www.infoadex.es/home/estudio-infoadex-de-la-inversion-publicitaria-en-espana-2019/

Key, T. M. (2017). Domains of Digital Marketing Channels in the Sharing Economy. Journal of Marketing Channels, 24(1-2), 27-38. https://doi.org/10.1080/1046669X.2017.1346977

Launchmetrics Content Team. (2015, January 19). La Pirámide del Influencer Engagement: los 3 tipos de influencia (The Pyramid of Influencer Engagement: the 3 types of influence). LaunchMetrics. https://www.launchmetrics.com/es/recursos/piramide-influencer-engagement

Lee, J., Sudarshan, S., Sussman, K., Bright, L., \& Eastin, M. (2021). Why are consumers following social media influencers on Instagram? Exploration of consumers' motives for following influencers and the role of materialism. International Journal of Advertising. https://doi.org/10.1080/02650487.2021.1964226

López Herrera, Á. (2016, June 25). ¿Qué requisitos debe tener un influencer? (What requirements must an influencer have?). Álvaro López Herrera. https://alvarolopezherrera.com/querequisitos-debe-tener-un-influencer/

López-Sáez, M. \& Bustillos, A. (2008). Effectiveness of social influence techniques in social marketing advertising. International Journal of Social Psychology, 23(1), 17-28. thttps://doi.org/10.1174/021347408783399525

Martín, S. (2019). Ranking de Influencer de Viajes en España. Metricool. https://metricool.com/es/influencers-viajes-espana/

Martínez-Sala, A. M., Monserrat-Gauchi, J., \& Segarra-Saavedra, J. (2019). El influencer 2.0 turístico: de turista anónimo a líder de opinion (The influencer tourist 2.0: from anonymous tourist to opinion leader). Revista Latina de Comunicación Social, (74), 1345-1348. https://doi.org/10.4185/RLCS-2019-1388 
Navas, C. (2018, March 1). 4 objetivos de una campaña de influencer marketing (4 objectives of an influencer marketing campaign). Flocker. https://flockler.com/es/blog/4-objetivosde-una-campana-de-influencer-marketing

Núñez, V. (2018, April 9). ¿Qué es el marketing de contenidos? (What is content marketing?). https://vilmanunez.com/que-es-el-marketing-de-contenidos/

Peñalver, P. (2019). Marca personal: el caso de los influencers (Personal brand: the case of influencers) (Undergraduate thesis, Universidad Pontificia Comillas). https://repositorio.comillas. edu/xmlui/bitstream/handle/11531/27148/TFG-\%20PeAalver\%20HernAndez\%2C\%20 Paloma.pdf?sequence=lyisAllowed=y Pérez, M. (2016). Influencer engagement, una estrategia de comunicación que conecta con la generación millenial (Influencer engagement, a communication strategy that connects with the millennial generation) (Undergraduate thesis, Universidad de Alicante). https://rua.ua.es/dspace/bitstream/10045/57327/1/ Estrategia_de_la_publicidad_y_de_las_relaciones_publi_PEREZ_CONDES_MONICA. $\underline{\mathrm{pdf}}$

Pop, R., Săplăcan, Z., Dabija, D., \& Alt, M. (2021). The impact of social media influencers on travel decisions: The role of trust in consumer decision journey. Current Issues in Tourism. https://doi.org/10.1080/13683500.2021.1895729

Rentero, C. (2018). Los 4 pilares del éxito del marketing digital (The 4 pillars of digital marketing success). Tecnohotel: revista profesional para la hostelería y restauración, (479), 44-45.

Ruiz, R. (2020, 24 de marzo). Diez canales de YouTube para evadirse viajando sin salir de casa (Ten YouTube channels to escape traveling without leaving home). eldiario.es. https://www.eldiario.es/viajes/guias_y_consejos/canalesYouTube-evadirse-viajandosalir_0_1009300154.html

Santamaría, E. \& Meana, R. (2017). Redes sociales y "fenómeno influencer". Reflexiones desde una perspectiva psicológica (Social media and "influencers". Reflections from a psychological perspective). Miscelánea Comillas, 75(147), 445-447.

https://revistas.comillas.edu/index.php/miscelaneacomillas/article/view/8433

Santiago, A. (2015, December 15). Los influencers como prescriptores de marca (Influencers as brand prescribers). The Mood Project. Friendly Branding. http://themoodproject.com/blog/los-influencers-como-prescriptores-de-marca/

Sixto-García, J., López-García, X., \& Gómez de la Fuente, M. del C. (2021). Instant messaging as an informative source in organizational communication: WhatsApp Business in Mexico and Spain. Comunicación Y Sociedad, 1-26. https://doi.org/10.32870/cys.v2021.7679

Suárez, S. (2021). El 'marketing' de influencia y las marcas relevantes (Influence marketing and relevant brands). Harvard Deusto Business Review, (308), 24-31.

https://www.harvard-deusto.com/el-marketing-de-influencia-y-las-marcas-relevantes

Ure, M. (2018). Engagement estratégico y encuentro conversacional en los medios sociales (Strategic engagement and conversational encounter on social media). Revista de Comunicación, 17(1), 182-190. https://doi.org/10.26441/RC17.1-2018-A10

Yllmaz, M., Sezerel, H., \& Uzuner, Y. (2020). Sharing experiences and interpretation of experiences: A phenomenological research on Instagram influencers. Current Issues in Tourism, 23(24), 3034-3041. https://doi.org/10.1080/13683500.2020.1763270 


\section{SOBRE LOS AUTORES}

PAULA RODRíGUez VALDERREy, es graduada en Periodismo por la Universidad de Santiago de Compostela y posee un Máster en Gestión Publicitaria por la Universidad Complutense de Madrid. Trabaja como responsable de comunicación interna en Sitel Spain.

iD https://orcid.org/0000-0002-7709-8785

JOSÉ SIXTO-GARCíA, es profesor del Departamento de Ciencias de la Comunicación de la Universidad de Santiago de Compostela. Fue director de Instituto de Medios Sociales (2013-2019). Sus líneas de investigación son los nuevos escenarios digitales y los modelos de redes. Entre sus publicaciones destacamos libros como Gestión profesional de redes sociales. Rutinas y estrategias de Community Management (2018) o artículos como "Co-creation in North American and European digital native media: Web, social networks and offline spaces", publicado en Journalism (2020).

(iD) https://orcid.org/0000-0002-2988-0975 